\title{
CORRIGENDUM
}

\section{Targeting gene expression to cones with human cone opsin promoters in recombinant $A A V$}

AM Komáromy, JJ Alexander, AE Cooper, VA Chiodo, LG Glushakova, GM Acland, WW Hauswirth and GD Aguirre

Gene Therapy (2008) 15, 1073; doi:10.1038/gt.2008.107

Correction to: Gene Therapy (2008) 15, 1049-1055; doi:10.1038/gt.2008.32

The authors would like to apologize for missing out the name of the author LG Glushakova of the University of Florida, Hauswirth Lab.

The correct author list is now shown above. 